Published online 2017 April 13.

\title{
Brain Near-Infrared Spectroscopy (NIRS)
}

\section{Soheila Refahi," and Hakimeh Saadati}

"Corresponding author: Soheila Refahi. E-mail: soheila52@yahoo.com

Received 2016 December 21; Accepted 2017 February 08.

\begin{abstract}
Near-infrared spectroscopy (NIRS) is a spectroscopic method that uses the near-infrared region of the electromagnetic spectrum. Typical applications include medical and physiological diagnostics. NIRS can be used for non-invasive assessment of brain function through the intact skull in human subjects by detecting changes in blood hemoglobin concentrations associated with neural activity, e.g., in branches of cognitive psychology as a partial replacement for fMRI techniques. NIRS cannot fully replace fMRI because it can only be used to scan cortical tissue, where fMRI can be used to measure activation throughout the brain. In this approach we compared NIRS with different functional brain tests.
\end{abstract}

This is an abstract presented in the 33rd Iranian congress of radiology (ICR) and the 15th congress of Iranian radiographic science association (IRSA). 